\title{
Effect of charged metal nanoparticles on carrier injection in graphene by an external electric field
}

\author{
Manaho Matsubara* and Susumu Okada ${ }^{\dagger}$ \\ Graduate School of Pure and Applied Sciences, University of Tsukuba, 1-1-1 Tennodai, Tsukuba, Ibaraki \\ 305-8571, Japan
}

\begin{abstract}
First-principles total-energy calculations clarified the effect of charged Al nanoparticles on carrier accumulation in graphene by an external electric field. Carrier injection in graphene with Al nanoparticles is sensitive to the relative position of the Al nanoparticles to the gate electrode. The nanoparticles sandwiched between graphene and electrode prevent the carrier injection in graphene, while the nanoparticles adsorbed on the opposite side do not affect the Dirac point shift, resulting in the successive carrier injection in graphene. Because of the density of state difference, the capacitance of graphene with Al nanoparticle also depends on the electrode position.
\end{abstract}

Graphene has been keeping a premier position not only in the low-dimensional sciences but also in the electronic device engineering due to its unique structural and electronic properties. ${ }^{1-8)}$ For the fundamental and applied sciences of graphene, it is mandatory to precisely control their electronic structure by external conditions, such as chemical modification, atom/molecule doping, and external electric field. In particular, the band gap engineering and the Fermi level tuning are highly important for fundamental study and application of graphene. In practical application of graphene, graphene inherently possesses hybrid structures with foreign materials in its device structures, such as insulating substrate, metal electrode, and atoms/molecules, which affect the electronic structure of graphene. ${ }^{11-18)}$ It has been demonstrated that graphene physically adsorbed on insulating substrates does not possesses the Dirac cone but quadratic dispersion band with a finite energy gap which depends on the surface morphology and atom species. ${ }^{12-15)}$ Atoms and molecules adsorbed on the graphene also modulate the Dirac cone of graphene by covalent and van der Waals interaction between adsorbates and graphene. ${ }^{16-18)}$ Although the carrier accumulation in pristine graphene and graphite under an external electric field has been steadily elucidated, ${ }^{19,20)}$ the micro-

\footnotetext{
*E-mail address: mmatsubara@comas.frsc.tsukuba.ac.jp

${ }^{\dagger}$ E-mail address: sokada@comas.frsc.tsukuba.ac.jp
} 
scopic mechanism of the carrier injection into graphene under such hybrid structures is not elucidated yet. In particular, for further advancing the device applications, it is important to unravel whether the foreign materials prevent or assist the carrier injection into graphene in the hybrid structures.

In this paper, we aim to give theoretical insight into the electronic properties of graphene adsorbing metal nanoparticles under the carrier injection by the external electric field, using first-principles total energy calculations combined with the effective screening medium method within the framework of the density functional theory. Our calculations show that the carrier injection is prevented by the metal nanoparticles adsorbed on the surface of graphene to the electrode, leading to the almost constant energy of the Dirac point of graphene with respect to the carrier species and concentration. In contrast, for the case that the metal nanoparticles are adsorbed on the opposite of the graphene, the eigen value of the Dirac point monotonically shifts downward with increasing the number of electron, indicating that the metal nanoparticles do not affect the carrier injection in graphene by the electric field. The present results provide the guiding principle for designing graphene based electronic devices containing charged nanoparticles.

All calculations were performed using the density functional theory (DFT) ${ }^{21,22)}$ with a Simulation Tool for Atom TEchnology (STATE) package. ${ }^{23)}$ To express the exchange correlation potential among the interacting electrons, the local density approximation is applied with the Perdew-Wang functional form fitting to the Quantum Monte Carlo results on the homogeneous electron gas. ${ }^{24,25)}$ We use ultrasoft pseudopotentials generated by the Vanderbilt scheme for describing the interaction between nuclei and electrons. ${ }^{26)}$ The valence wave functions and charge density were expanded in terms of the plane-wave basis set with cutoff energies of 25 and 225 Ry, respectively. The Brillouin-zone integration was performed with the $\Gamma$-centered $4 \times 4 \times 1$ uniform $\boldsymbol{k}$-mesh for self-consistent electronic structure calculations for the graphene with $4 \times 4$ lateral periodicity, which corresponds to the $16 \times 16 \times 1 \boldsymbol{k}$-mesh in a primitive $1 \times 1$ cell of graphene, resulting in sufficient convergence for the geometric and electronic structures. All atoms were fully optimized until the remaining force acting on each atom was less than $0.005 \mathrm{Ry} / \AA$ under a fixed lateral lattice constant of $9.83 \AA$ corresponding to the experimental value of $4 \times 4$ lateral cell of graphene.

In the present work, we consider the graphene field effect transistor (FET) in which $\mathrm{Al}$ nanoparticles are physorbed on the graphene surfaces. $\mathrm{Al}_{4}$ and $\mathrm{Al}_{3}$ with square and 
(a)

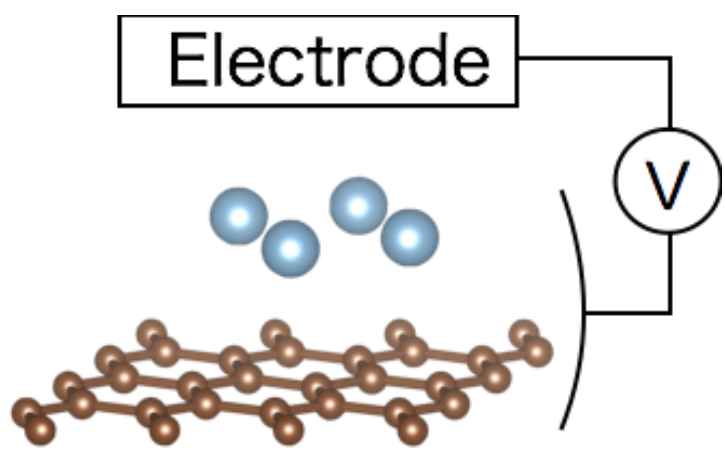

(b)

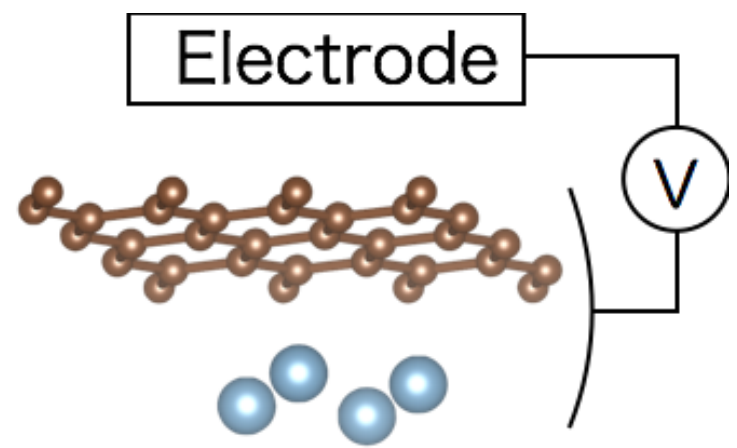

Fig. 1. Structural model of graphene FET adsorbing Al nanoparticles with (a) the electrode-Al-graphene and (b) the electrode-graphene-Al arrangements. Small and large balls denote $\mathrm{C}$ and $\mathrm{Al}$ atoms, respectively.

triangular shapes, respectively, are adsorbed on graphene per $4 \times 4$ lateral periodicity. The Al nanoparticles are adsorbed in parallel arrangement with the optimum spacing of 3.2 and $3.6 \AA$ for $\mathrm{Al}_{4}$ and $\mathrm{Al}_{3}$, respectively. To investigate the electronic properties of graphene with metal nanoparticles under the electron and hole injection, we adopted the effective screening medium (ESM) method to solve the Poisson equation under the boundary condition in which a planar metal electrode and half-infinite vacuum are situated at two cell boundaries parallel to the graphene layer (Fig. 1). ${ }^{27)}$ Electron and hole were injected by the planar gate electrode situated at the cell boundary with the vacuum spacings of about $4.5 \AA$ to graphene or Al particles, mimicking the graphene FET with the top and bottom gate electrodes with respect to the $\mathrm{Al}$ nanoparticle (Fig. 1). The electrode is simulated by an effective screening medium with an infinite relative permittivity. During the calculations under a finite electric field, the atomic structure of the hybrid consisting of graphene and $\mathrm{Al}$ nanoparticules are fixed as the optimized structure obtained under the zero electric field.

Figures 2(a) and 2(b) show the electronic energy band of graphene adsorbing $\mathrm{Al}_{4}$ and $\mathrm{Al}_{3}$ under the zero electric field, respectively. Three and two less dispersive bands emerge just above the Dirac cone for $\mathrm{Al}_{4}$ and $\mathrm{Al}_{3}$, respectively, associated with the electron states of $\mathrm{Al}$ nanoparticle. The wave function analysis indicates that these states possess the highest occupied state of an isolated $\mathrm{Al}$ nanoparticle with bonding $p$ states nature. Because the $E_{F}$ is located above the Dirac cone, the electron is transferred from $\mathrm{Al}$ nanoparticles to graphene. In accordance with this charge transfer, the Al nanoparticles possess partially filled electron states at the $E_{F}$ and act as charged impurities for carrier 


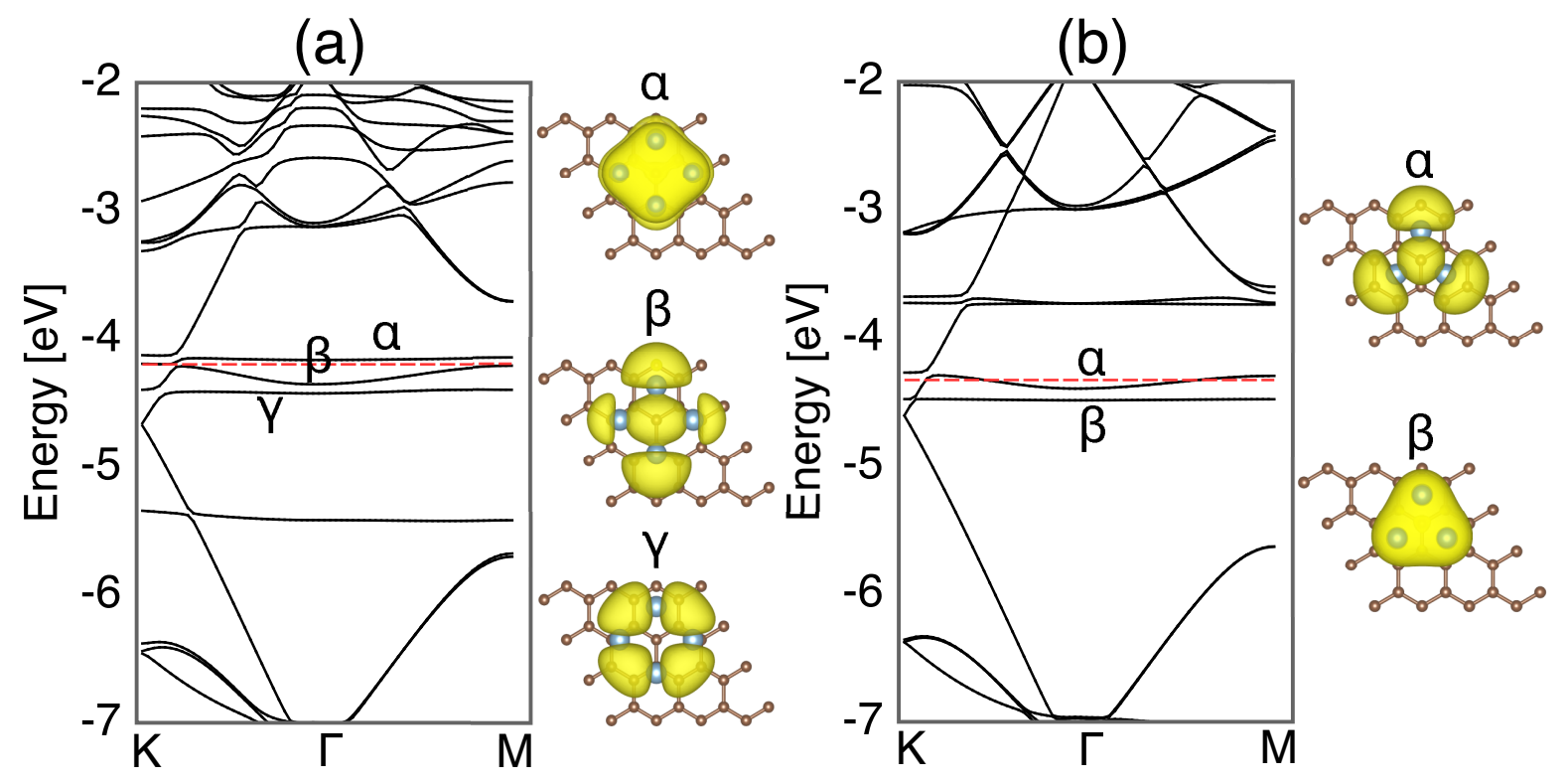

Fig. 2. Electronic energy band of graphene adsorbing (a) $\mathrm{Al}_{4}$ and (b) $\mathrm{Al}_{3}$ nanoparticles together with the isosurfaces of the squared wavefunctions labelled in left panels. The dotted red lines denote the Fermi level. The energies are measured from that of the vacuum level.

injection into graphene. The partially filled flat dispersion bands cause large density of states at the $E_{F}$ and are expected to prevent electrons injection in graphene by the external electric field.

Under the external electric field by the gate electrode, injected carriers are primary accommodated in graphene or $\mathrm{Al}$ nanoparticles which are situated at the electrode side: The injected electron and hole are distributed on $\mathrm{Al}$ nanoparticles in the electrode-Algraphene arrangement, while they are distributed on graphene layer in the electrodegraphene-Al arrangement (Fig. 3). In addition to the carrier density at the electrode side, the carriers are slightly spilled out the other side of the electrode for the hole doping in the electrode-graphene-Al arrangement [the right panels in Figs. 3(b) and $3(\mathrm{~d})]$. The fact indicates that the carrier accumulation in graphene is sensitive to the relative arrangement of the metal nanoparticles to the electrode: Metal nanoparticles situated between graphene and electrode may prevent carrier injection into graphene by the electric field.

Figure 4 shows the electronic energy band of graphene adsorbing $\mathrm{Al}_{4}$ and $\mathrm{Al}_{3}$ under the external electric field that injects one electron or hole in the hybrid systems. The electronic structure of the hybrids under the electric field is sensitive to the carrier species, carrier concentration, and relative arrangement of the metal nanoparticles to 
(a)

(b)
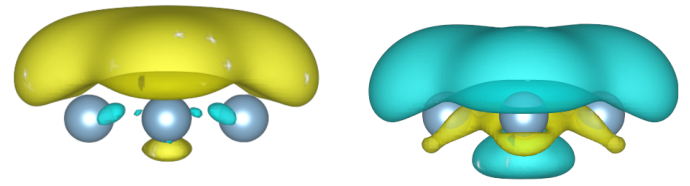

$\infty=\infty=\infty=\infty$

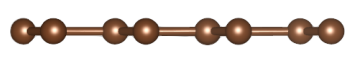

(c)
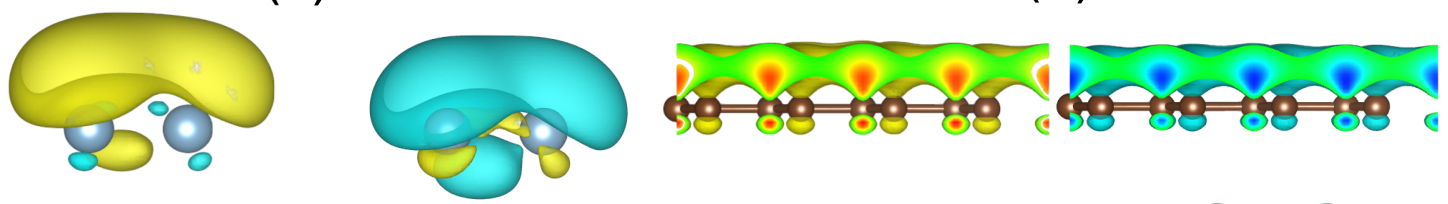

$\infty=\infty=\infty=\infty$
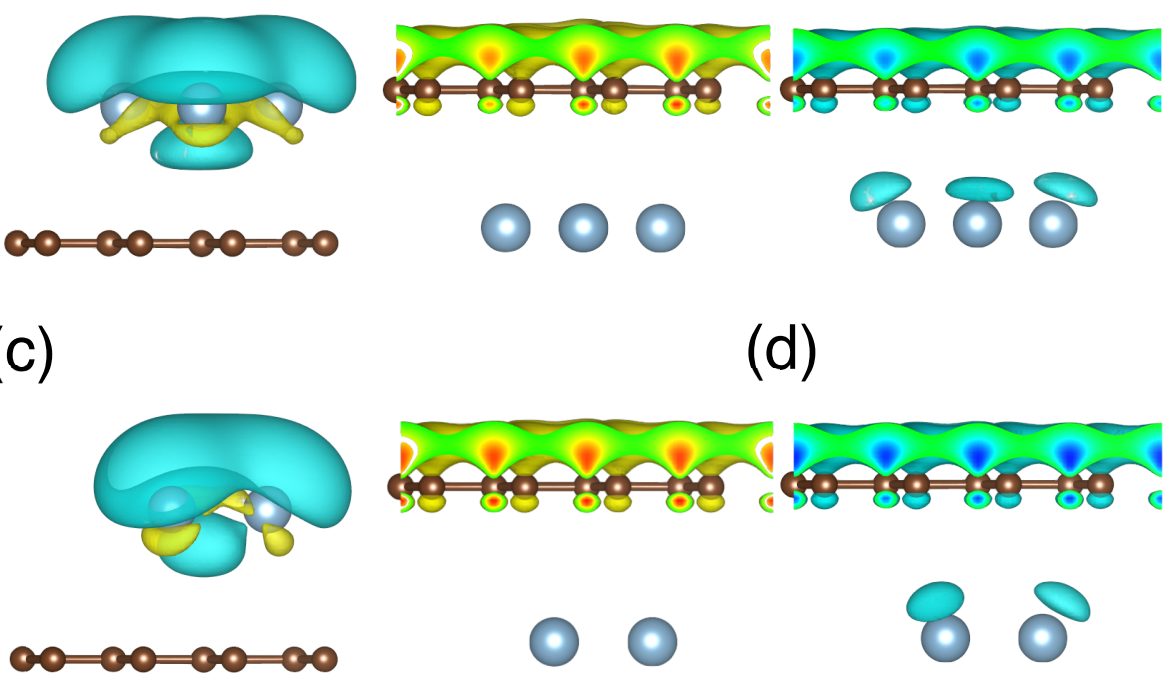

.

(d)

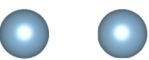

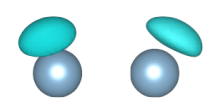

Fig. 3. Isosurfaces of the distribution of the accumulated electron and hole by the gate electrode in graphene- $\mathrm{Al}_{4}$ hybrids in which the $\mathrm{Al}_{4}$ is adsorbed (a) above and (b) below the graphene layer. Isosurfaces of the distribution of the accumulated one electron and hole by the gate electrode in graphene- $\mathrm{Al}_{3}$ hybrids in which the $\mathrm{Al}_{3}$ is adsorbed (c) above and (d) below the graphene layer. In each figure, the left and right panels show the isosurfaces under one electron and hole doping, respectively. Yellow and blue isosurfaces denote the region where the electron and hole increase, respectively. Small and large balls denote $\mathrm{C}$ and $\mathrm{Al}$ atoms, respectively. The electrode is situated at the top of each figure with the vacuum spacing of about $4.5 \AA$ to Al nanoparticle or graphene.

the electrode, reflecting the characteristic distribution of the accumulated carriers. On the other hand, the electronic structure weakly depends on the metal nanoparticle size except the number of flat bands associated with the metal nanoparticles. The Dirac point of graphene is insensitive to the hole and electron injections for the case that the metal nanoparticles are situated between the graphene layer and the metal electrode. In the case, flat dispersion bands associated with the metal nanoparticles sensitively shift upward and downward for the hole and electron doping, respectively. Therefore, the carrier injection into graphene rarely occurs. On the other hand, the Dirac point exhibits upward and downward shift under the hole and electron doping, respectively, for the case that the metal nanoparticles are located at the opposite surface of the graphene to the electrode, indicating that the carrier injection into graphene is possible under the metal nanoparticle arrangement. In contrast to the Dirac point, the flat band states associated with $\mathrm{Al}$ nanoparticles retain their energy value during the carrier injection. 
(a)

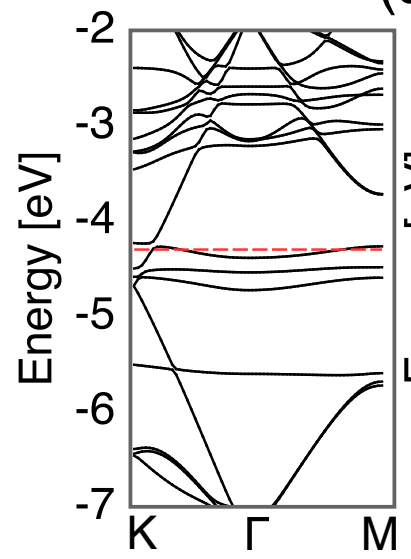

(c)

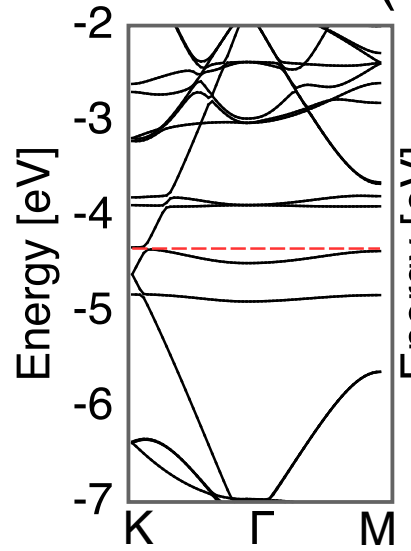

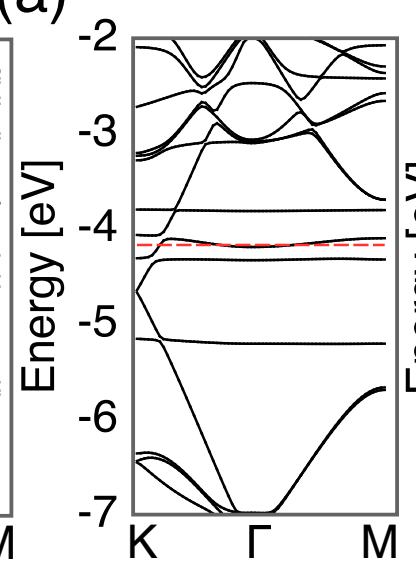

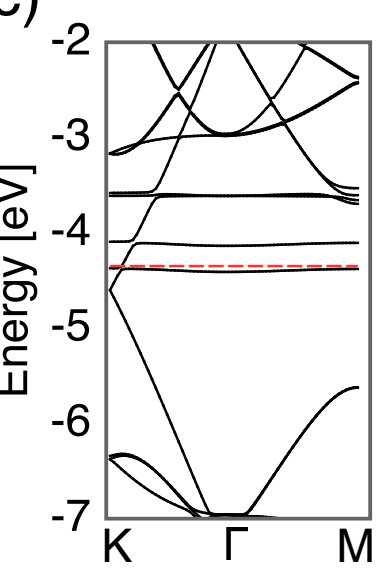

(b)
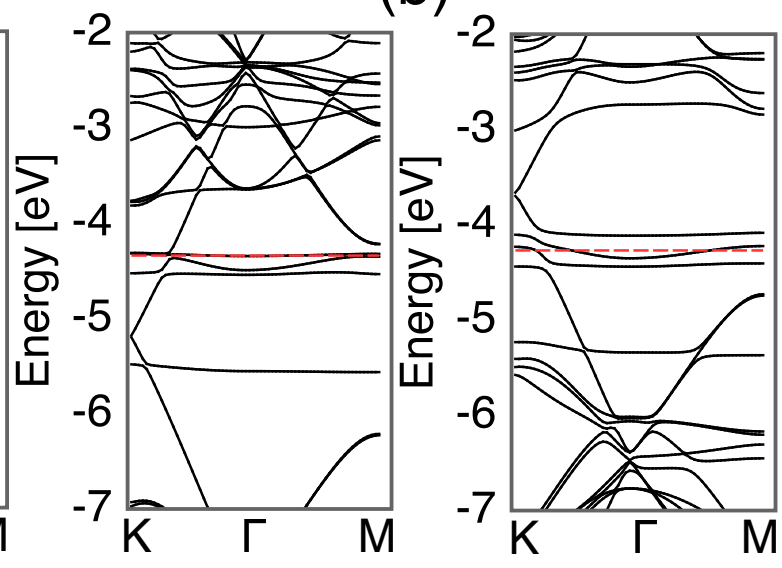

(d)

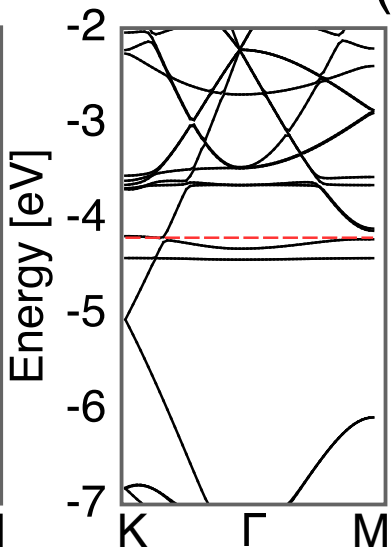

Fig. 4. Electronic energy band of graphene adsorbing $\mathrm{Al}_{4}$ nanoparticles adsorbed (a) above and (b) below the graphene layer. Electronic energy band of graphene adsorbing $\mathrm{Al}_{3}$ nanoparticles adsorbed (c) above and (d) below the graphene layer. In each figure, the left and right panel shows the energy band under one electron and hole doping, respectively. The dotted red lines denote the Fermi level. The energies are measured from that of the vacuum level.

Figure 5 shows the eigen value of the Dirac point of graphene adsorbing Al nanoparticles as a function of the carrier concentration. The Dirac point almost retains the energy of about $-4.6 \mathrm{eV}$ for all carrier concentration between 1 hole and 1 electron under the metal arrangement in which metal nanoparticles are situated between graphene and electrode, indicating that any carriers are rarely injected into graphene by the external electric field. In the case, instead of the almost constant eigen value of the Dirac point, the electron states associated with the metal nanoparticle substantially shift downward with increasing the number of electron. Injected electrons stabilize the electronic states of metal nanoparticle, because the flat band states of $\mathrm{Al}$ nanoparticles possess bonding $p$ state nature (Fig. 2). In contrast, the Dirac point monotonically shifts downward 
(a)

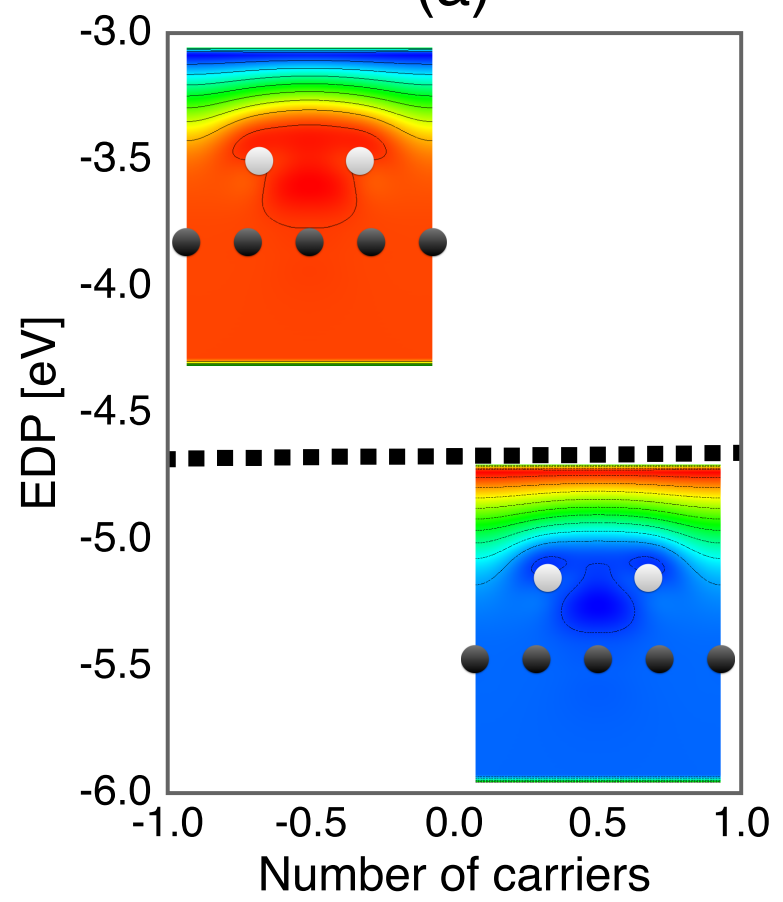

(c)

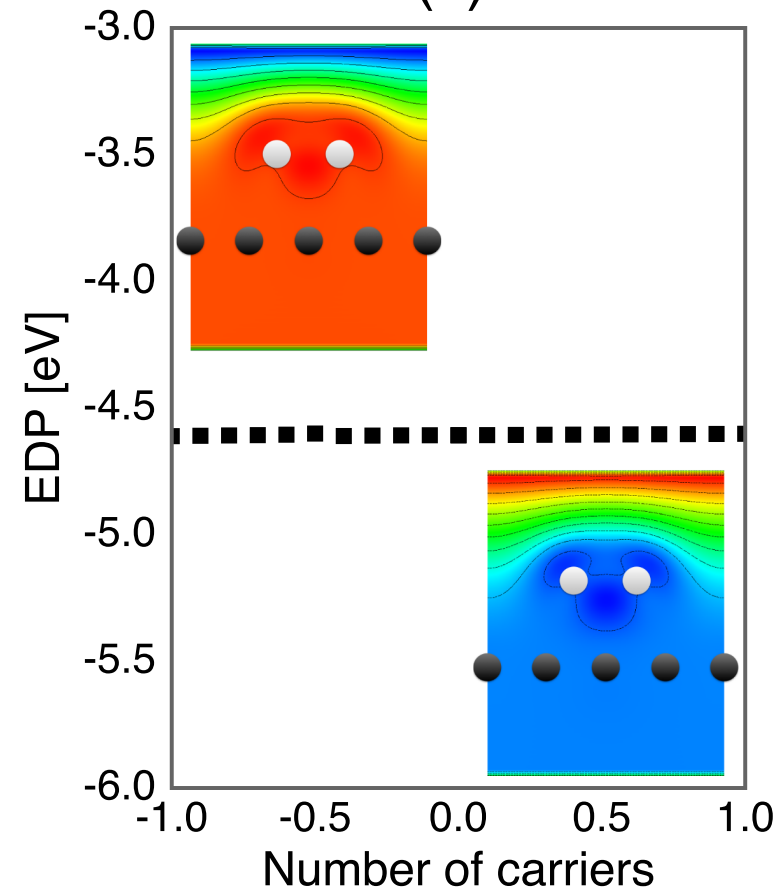

(b)

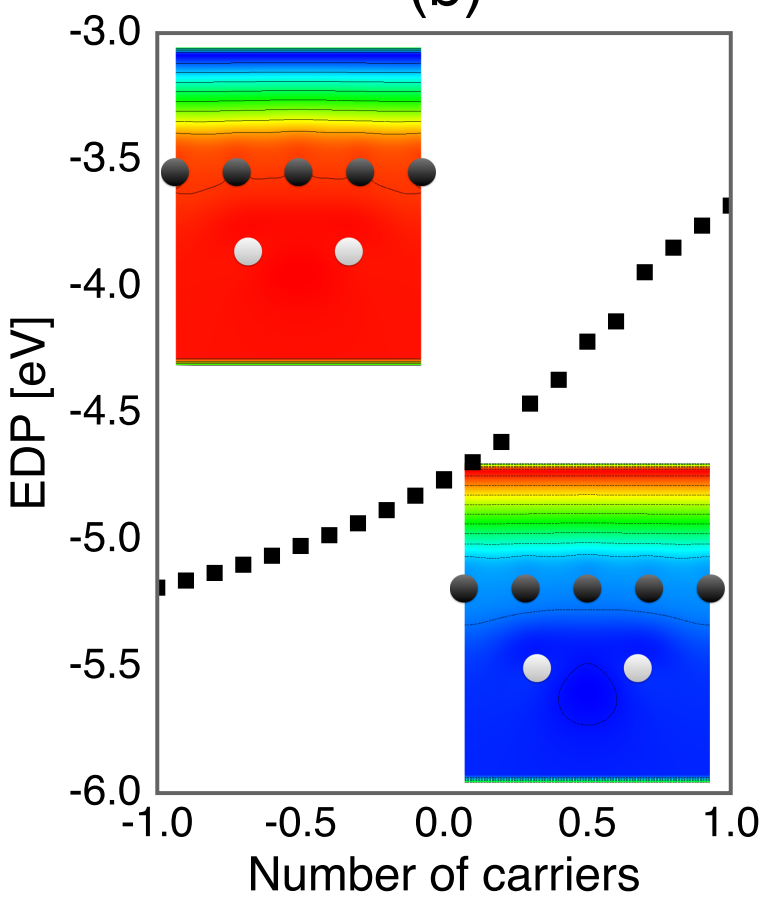

(d)

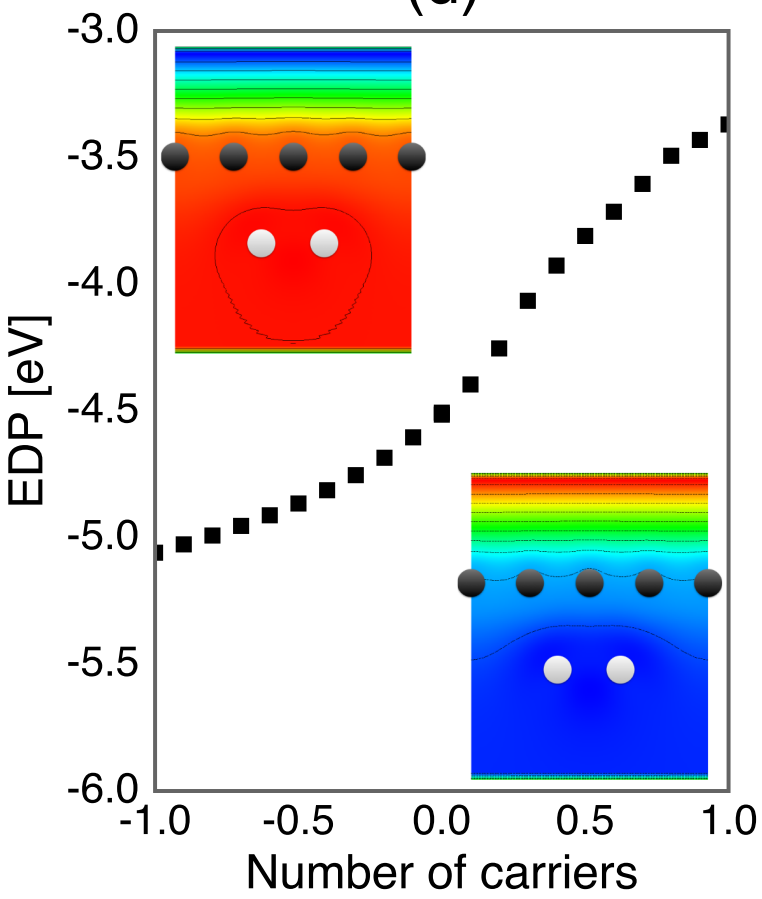

Fig. 5. Eigen value of the Dirac point (EDP) as a function of the injected carrier for the graphene- $\mathrm{Al}_{4}$ hybrid in which $\mathrm{Al}_{4}$ are adsorbed (a) above and (b) below the graphene layer. EDP as a function of the injected carrier for the graphene- $\mathrm{Al}_{3}$ hybrid in which $\mathrm{Al}_{3}$ are adsorbed (c) above and (d) below the graphene layer. Negative and positive values denote the number of the injected electron and hole, respectively. The energies are measured from that of the vacuum level. Upper and lower Insets in each panel indicate the contour plots of the electrostaitc potential of graphene-Al hybrids under carrier concentration of -1.0 and 1.0, respectively. Dark and pale shaded circles denote $\mathrm{C}$ and $\mathrm{Al}$ atoms, respectively. 
with increasing the number of electrons under the metal arrangement in which metal nanoparticles are adsorbed on the opposite surface of graphene to the electrode. It should be noted that the electric field is mostly screened by the Al or graphene situated at the electrode side (insets of Fig. 5), although Al nanoparticles and graphene have nanometer size and atomic thickness, respectively. This field profile leads to the fact that $\mathrm{Al}$ nanoparticles hardly affect the carrier injection into the graphene for the electrode-graphene-Al arrangement. These facts give guiding principles for designing and controlling physical properties of graphene-based FET: To control the Fermi level of the graphene FET, it is necessary to exclude charged nanoparticles from the space between graphene and electrode.

Finally, we check the total capacitance of graphene adsorbing metal nanoparticles as a function of the number of carriers, in which the geometrical and quantum capacitance are fully taken into account. The total capacitance is evaluated using a formula $C=\frac{d Q}{d \mu}=\frac{C_{0} D}{C_{0}+D}$ where $C_{0}$ and $D$ denote the geometrical capacitance and density of states of Al-graphene hybrids, respectively. ${ }^{28,29)}$ Note that the potential difference $d \mu$ is evaluated by taking the difference between the electrostatic potential at two cell boundaries (the potential at the electrode and vacuum). Therefore, the capacitance calculated here is slightly underestimated compared with the actual value. The capacitance depends on the size of $\mathrm{Al}$ nanoparticles, relative arrangements of the $\mathrm{Al}$ nanoparticle to the gate electrode, and the carrier concentration (Fig. 6). The capacitance of the electrode-Al-graphene arrangement is higher than that of the electrode-graphene- $\mathrm{Al}$ arrangement, for all carrier concentration except the small carrier concentration and for both nanoparticles. The higher capacitance of the electrode-Al-graphene arrangement is ascribed to the large density of states (DOS) associated with the partially filled flat band states of metal nanoparticle at the Fermi level adsorbed on graphene. The van Hove singularity of the metal nanoparticle results in the geometric capacitance $C_{0}$ which is the largest limit of the capacitance. On the other hand, the small capacitance of the electrode-graphene-Al arrangement is caused by the linear DOS near the Fermi level associated with the Dirac cone, even though the arrangement has larger contact area with the electrode, resulting in the large geometrical capacitance.

In summary, based on DFT combined with the ESM method, we have studied the electronic structure of graphene adsorbing $\mathrm{Al}$ nanoparticles per $4 \times 4$ lateral cell. The Al nanoparticles affect carrier injection in graphene with respect to their mutual arrangement to the electrode: The electron and hole injections are prevented by the 
(a)

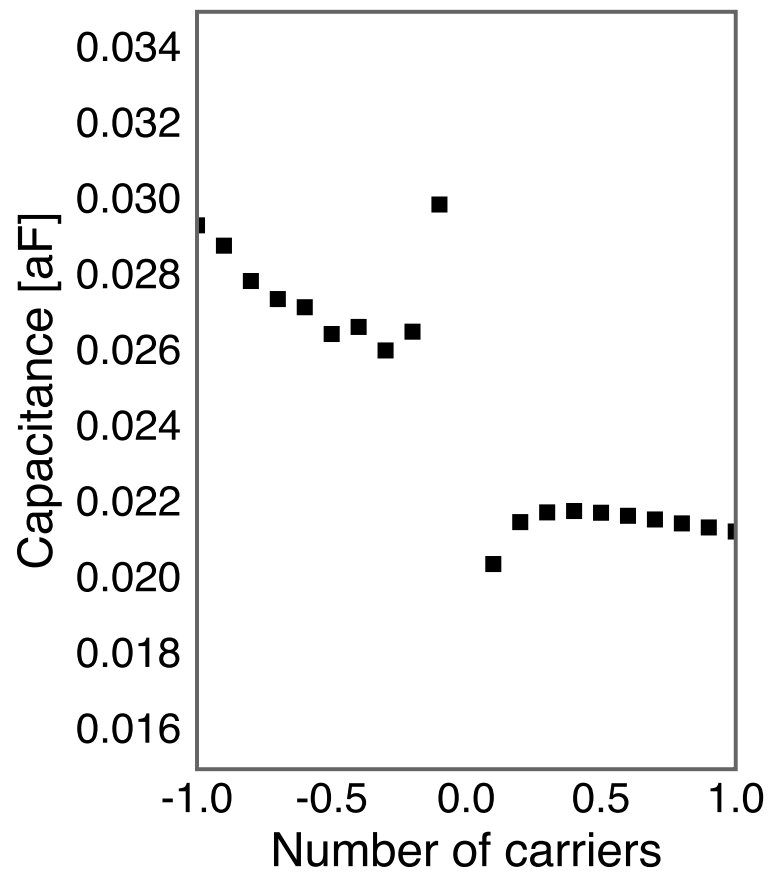

(c)

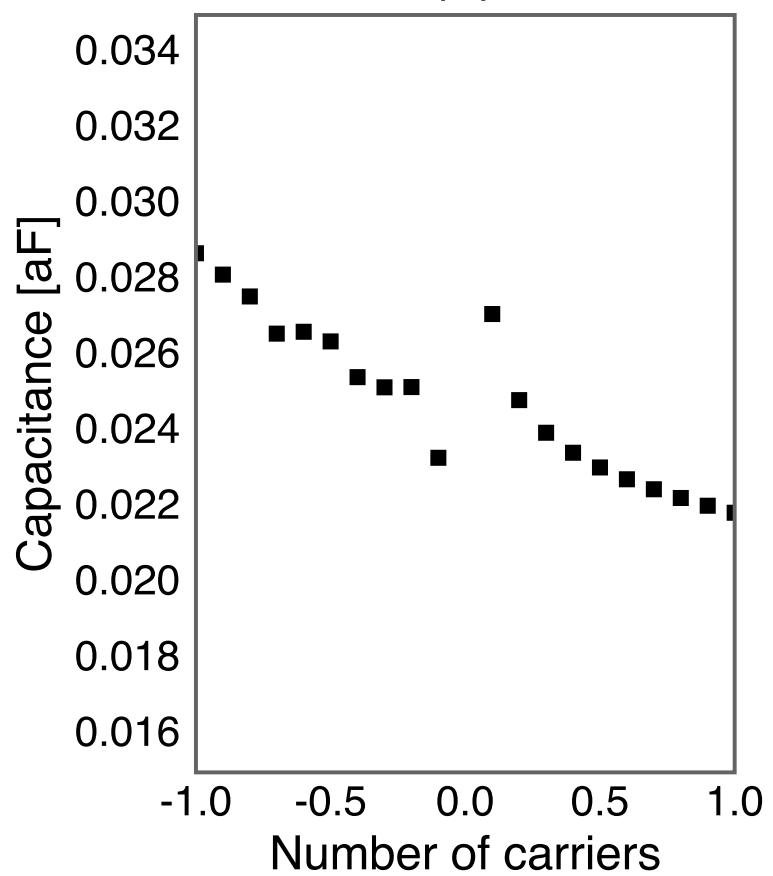

(b)

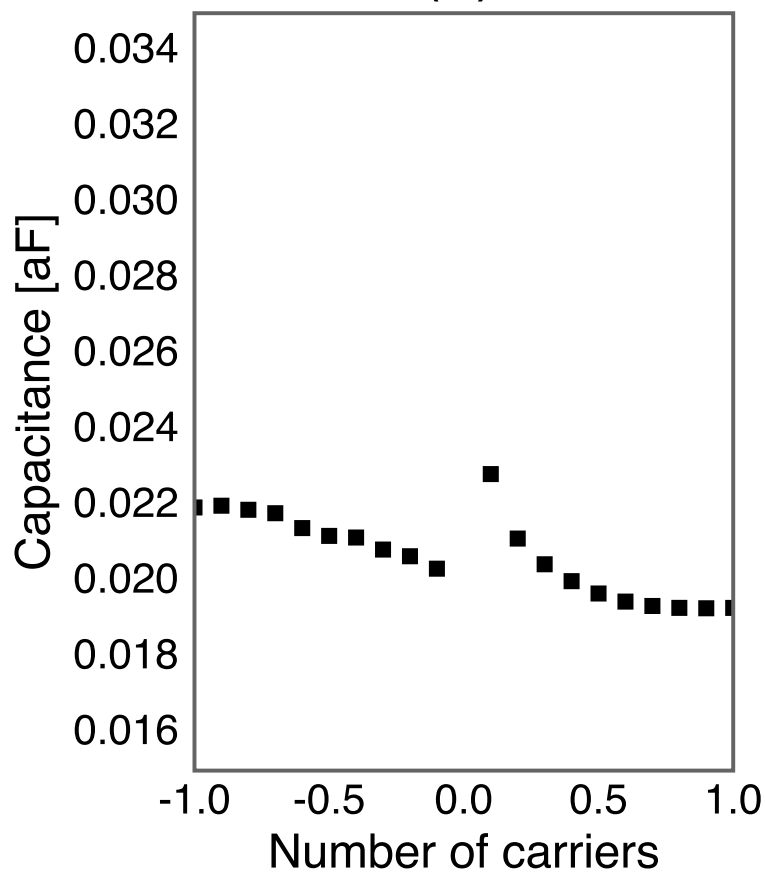

(d)

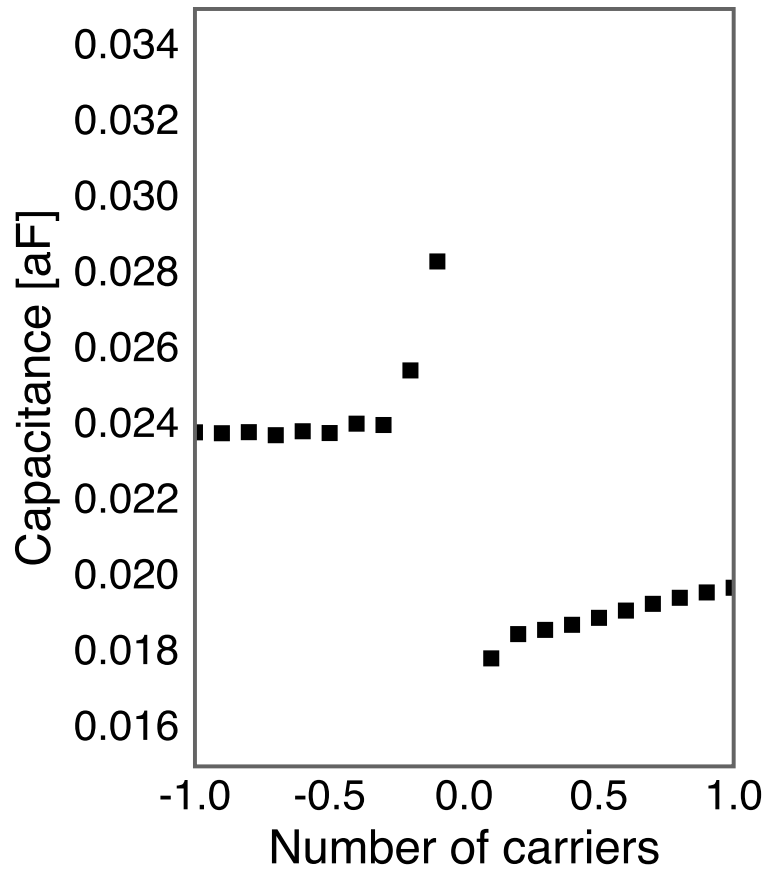

Fig. 6. Capacitance per $4 \times 4$ lateral cell of the graphene- $\mathrm{Al}_{4}$ hybrid in which $\mathrm{Al}_{4}$ are adsorbed (a) above and (b) below the graphene layer. Capacitance per $4 \times 4$ lateral cell of the graphene- $\mathrm{Al}_{3}$ hybrid in which $\mathrm{Al}_{3}$ are adsorbed (c) above and (d) below the graphene layer. Negative and positive values denote the number of the injected electron and hole, respectively. 
metal nanoparticles adsorbed on the surface of graphene to the electrode, leading to the constant energy of the Dirac cone of graphene. In contrast, for the case that the Al nanoparticles are adsorbed on the opposite surface of graphene to the electrode, the Dirac point monotonically shifts downward with injecting electron, indicating that the metal nanoparticles do not affect the carrier injection in graphene by the electric field. Our findings provide the guiding principle for designing graphene based electronic devices containing charged nanoparticles.

\section{Acknowledgements}

This work was supported by CREST, from the Japan Science and Technology Agency, JSPS KAKENHI Grant Numbers JP25246010, JP16H00898, and JP16H06331 from Japan Society For the Promotion of Science, and the Joint Research Program on ZeroEmission Energy Research, Institute of Advanced Energy, Kyoto University. Part of the calculations was performed on an NEC SX-Ace at the Cybermedia Center at Osaka University and on an SGI ICE XA/UV at the Institute of Solid State Physics, The University of Tokyo. 


\section{References}

1) M.S. Dresselhaus, G. Dresselhaus, Intercalation compounds of graphite, Adv. Phys. 30, 139 (1981).

2) A.H.C. Neto, F. Guinea, N.M.R. Peres, K.S. Novoselov, A.K. Geim, The electronic properties of graphene, Rev. Mod. Phys. 81, 109(2009).

3) K.S. Novoselov, A.K. Geim, S.V. Morozov, D. Jiang, Y. Zhang, S.V. Dubonos, I.V. Grigorieva, and A.A. Firsov, Science 306, 666 (2004).

4) K.S. Novoselov, A.K. Geim, S.V. Morozov, D. Jiang, M.I. Katsnelson, I.V. Grigorieva, S.V. Dubonos, and A.A. Firsov, Nature 438, 197 (2005).

5) Y. Zhang, Y.-W. Tan, H.L. Stormer, and P. Kim, Nature 438, 201 (2005).

6) K. I. Bolotin, K. J. Sikes, Z. Jiang, M. Klima, G. Fudenberg, J. Hone, P. Kim, and H. L. Stormer, Solid State Commun. 146, 351 (2008).

7) J.B. Oostinga, H.B. Heersche, X. Liu, A.F. Morpurgo, and L.M.K. Vandersypen, Nature Mater. 7151 (2007).

8) Y. Zhang, T.-T. Tang, C. Girit, Z. Hao, M.C. Martin, A. Zettl, M.F. Crommie, Y.R. Shen, and F. Wang, Nature 459, 820 (2009).

9) M. F. Craciun, S. Russo, M. Yamamoto, J. B. Oostinga, A. F. Morpurgo, and S. Tarucha, Nat. Nanotechnol. 4, 383 (2009).

10) K. S. Novoselov, A. K. Geim, S. V. Morozov, D. Jiang,1 Y. Zhang, S. V. Dubonos, I. V. Grigorieva, A. A. Firsov, Science 306, 666 (2004).

11) T. Ohta, A. Bostwick, T. Seyller, K. Horn, and E. Rotenberg, Science 313, 915 (2006).

12) S. Y. Zhou, G.-H. Gweon, A.V. Fedorov, P.N. First, W.A. de Heer, D.-H. Lee, F. Guinea, A.H.C. Neto, and A. Lanzara, Nature Mater. 6, 770 (2007).

13) A. Mattausch and O. Pankratov, Phys. Rev. Lett. 99, 076802 (2007).

14) N. T. Cuong, M. Otani, and S. Okada, Phys. Rev. Lett. 106, 106801 (2011).

15) K. Kamiya, N. Umezawa, and S. Okada, Phys. Rev. B 83, 153413 (2011).

16) M. Igami, S. Okada, and K. Nakada, Synthetic Metal 121, 1233 (2001).

17) P. Giannozzi, R. Car, and G. Scoles, J. Chem. Phys. 118, 103 (2003).

18) N.-T. Cuong, M. Otani, and S. Okada, Appl. Phys. Lett. 101, 233106 (2012).

19) M. Otani, Y. Takagi, M. Koshino, and S. Okada, Appl. Phys. Lett. 96, 242504 (2010).

20) M. Otani and S. Okada, J. Phys. Soc. Jpnt. 79, 073701 (2010). 
21) P. Hohenberg and W. Kohn, Phys. Rev. 136, B864 (1964).

22) W. Kohn and L. J. Sham, Phys. Rev. 140, A1133 (1965).

23) Y. Morikawa, K. Iwata, and K. Terakura, Appl. Surf. Sci. 11, 169 (2000).

24) D. M. Ceperley and B. J. Alder, Phys. Rev. Lett. 45, 566 (1980).

25) J.P. Perdew and Y. Wang, Phys. Rev. B 4513244 (1992).

26) D. Vanderbilt, Phys. Rev. B 41, 7892 (1990).

27) M. Otani and O. Sugino, Phys. Rev. B 73, 115407 (2006).

28) M. Büttiker, J. Phys.: Condens. Matter 5, 9361 (1993).

29) K. Uchida and S. Okada, Phys. Rev. B 76, 155436 (2007). 